\title{
Metabolic engineering of Saccharomyces cerevisiae for 7-dehydrocholesterol overproduction
}

\author{
Xiao-Jing Guo 1,2, Wen-Hai Xiao 1,2, Ying Wang ${ }^{1,2}$, Ming-Dong Yao ${ }^{1,2}$, Bo-Xuan Zeng 1,2, Hong Liu,2,
} Guang-Rong Zhao ${ }^{1,2}$ and Ying-Jin Yuan ${ }^{1,2^{*}}$

\begin{abstract}
Background: 7-Dehydrocholesterol (7-DHC) has attracted increasing attentions due to its great medical value and the enlarging market demand of its ultraviolet-catalyzed product vitamin $\mathrm{D}_{3}$. Microbial production of 7-DHC from simple carbon has been recognized as an attractive complement to the traditional sources. Even though our previous work realized 7-DHC biosynthesis in Saccharomyces cerevisiae, the current productivity of 7-DHC is still too low to satisfy the demand of following industrialization. As increasing the compatibility between heterologous pathway and host cell is crucial to realize microbial overproduction of natural products with complex structure and relative long pathway, in this study, combined efforts in tuning the heterologous $\triangle^{24}$-dehydrocholesterol reductase (DHCR24) and manipulating host cell were applied to promote 7-DHC accumulation.
\end{abstract}

Results: In order to decouple 7-DHC production with cell growth, inducible GAL promoters was employed to control 7-DHC synthesis. Meanwhile, the precursor pool was increased via overexpressing all the mevalonate (MVA) pathway genes (ERG10, ERG13, tHMG1, ERG12, ERG8, ERG19, IDI1, ERG20). Through screening DHCR24s from eleven tested sources, it was found that DHCR24 from Gallus gallus (Gg_DHCR24) achieved the highest 7-DHC production. Then 7-DHC accumulation was increased by $27.5 \%$ through stepwise fine-tuning the transcription level of Gg_DHCR24 in terms of altering its induction strategy, integration position, and the used promoter. By blocking the competitive path ( $\triangle E R G 6$ ) and supplementing another copy of Gg_DHCR24 in locus ERG6, 7-DHC accumulation was further enhanced by 1.07-fold. Afterward, 7-DHC production was improved by $48.3 \%$ (to $250.8 \mathrm{mg} / \mathrm{L}$ ) by means of deleting $N E M 1$ that was involved in lipids metabolism. Eventually, 7-DHC production reached to $1.07 \mathrm{~g} / \mathrm{L}$ in 5 - L bioreactor, which is the highest reported microbial titer as yet known.

Conclusions: Combined engineering of the pathway and the host cell was adopted in this study to boost 7-DHC output in the yeast. 7-DHC titer was stepwise improved by 26.9-fold compared with the starting strain. This work not only opens large opportunities to realize downstream de novo synthesis of other steroids, but also highlights the importance of the combinatorial engineering of heterologous pathway and host to obtain microbial overproduction of many other natural products.

Keywords: Metabolic engineering, 7-DHC, Host manipulation, DHCR24, Saccharomyces cerevisiae

\footnotetext{
*Correspondence: yjyuan@tju.edu.cn

${ }^{1}$ Key Laboratory of Systems Bioengineering (Ministry of Education),

School of Chemical \& Engineering, Tianjin University, No. 92, Weijin Road,

Nankai District, Tianjin 300072, People's Republic of China

Full list of author information is available at the end of the article
} 


\section{Background}

7-Dehydrocholesterol (7-DHC) is a high-valued sterol which can be directly converted into vitamin $\mathrm{D}_{3}$ under ultraviolet $B$ radiation [1]. Intake of adequate vitamin $D_{3}$ is not only essential to maintain musculoskeletal health, but also can reduce the risk of immune disorders, cardiovascular diseases, and many types of cancers $[2,3]$. Nowadays, many groups have recognized vitamin D deficiency as a worldwide public health problem, which has paved the way for a huge demand of vitamin $D_{3}$ or its direct precursor 7-DHC every year [2, 4]. Microbial production of 7-DHC from simple carbon (such as glucose) has been recognized as an attractive complement to the traditional sources by chemical synthesis and biotransformation [5]. Through blocking the endogenous ergosterol synthesis pathway (e.g., $\triangle E R G 5$ ) along with introducing the heterologous $\Delta^{24}$-dehydrocholesterol reductase (DHCR24) (Fig. 1a), heterologous production of 7-DHC has been successfully achieved in a safe (generally recognized as safe, GRAS) and robust host Saccharomyces cerevisiae [5-7]. However, the highest reported 7-DHC titer is $44.49 \mathrm{mg} / \mathrm{L}$ so far [7], which is still too low to satisfy the following industrialization process.

7-DHC biosynthesis pathway covers eight genes in mevalonate (MVA) pathway and nine genes in postsqualene pathway (Fig. 1a). In order to achieve an optimal output of the targeted pathway, it is preferred to engineer the pathway modules to balance the metabolic flux among these modules. Pathway engineering in terms of enlarging the precursor pools, blocking the competitive pathway, and introducing heterologous post-squalene pathway genes (i.e., ERG2,3 from Mus musculus), has been proven to be efficient to promote 7-DHC productivity [5-7]. However, the pathways are not isolated from the rest of cellular metabolism; in fact, they are tightly regulated by the endogenous system [8]. For instance, sterols accumulation is closely coupled to lipids synthesis in S. cerevisiae [9]. As reported by Fei et al. [10], sterols storage was upregulated by $70 \%$ in FLD1 (YLR404W, few lipid droplets gene1) deletion strain, along with the enlarged lipid droplets. It was also reported by Park et al. [11] that the loss of PAH1 (YMR165C, encoding phosphatidate phosphatase) led to striking changes in triacylglycerol and phospholipid metabolism, along with a significant increase on ergosterol synthesis. Therefore, manipulation of lipids metabolism to increase the flux flow toward sterol synthesis pathway would be helpful to improve 7-DHC accumulation in yeast. Besides that, introducing heterologous modules always upsets the original intracellular balance $[12,13]$. And heterologous sterols (such as campesterol) would bring cell burden via adhering or inserting to membrane structure [14-16]. In this case, decouple the cell growth from the product synthesis by employing inducible promoter [17] or regulating lipids metabolism to improve sterol storage [18] might alleviate this cell burden. To sum up, increasing the compatibility between heterologous pathway and host cell is crucial to realize microbial overproduction of heterologous chemicals. And in addition to pathway engineering, the settlements of some metabolic and regulatory issues within hosts also offer promising approaches to enhance product output. Thus, insufficient host engineering besides modification of pathway modules might be the reason for low 7-DHC titer in the previous works [5-7].

In this article, combined efforts in manipulating host and 7-DHC synthesis pathway were conducted to promote 7-DHC output base on our previous study [7] (Fig. 1b). On the one hand, decoupling 7-DHC production with the cell growth as well as deleting lipids metabolism gene(s) were adopted to apply host engineering. On the other hand, for pathway engineering, this work would mainly focus on tuning DHCR24 via screening enzyme sources and adjusting its transcriptional level. Consequently, combinatorial engineering of the heterologous enzyme and the host cell achieved 26.9-fold enhancement on 7-DHC output (to $1.07 \mathrm{~g} / \mathrm{L}$ ), which highlights the importance of this combinatorial engineering strategy to improve the compatibility between heterologous pathway and host cell for microbial overproduction of desired products.

\section{Results and discussion}

Preparing a modified yeast beneficial for 7-DHC synthesis

In order to guarantee sufficient precursor supply, all the functional genes in MVA pathway (Fig. 1a) were overexpressed according to Westfall et al. [17], i.e., supplementing one copy of ERG10, ERG13, ERG12, ERG8, ERG19, IDI1, and ERG20 as well as three copies of $t H M G 1$ (Fig. 1c). Meanwhile, to decouple 7-DHC production with cell growth, the constitutive promoters employed in the previous study were replaced by inducible GAL promoters to control the expression of the only heterologous gene (DHCR24) as well as the overexpressed MVA pathway genes (Fig. 1c, d), generating the strain SyBE Sc01130007 (Table 1 and Fig. 1b). Consequently, the whole fermentation process could be divided into glucose consumption phase (before GAL induction) and ethanol consumption phase (after GAL induction).

Moreover, our previous study has demonstrated that blocking competitive ergosterol biosynthesis pathway was essential for 7-DHC accumulation in yeast [7]. Accordingly, gene ERG5 was knocked out to block the metabolic flux to ergosterol (Fig. 1a), obtaining strain SyBE_Sc0125XJ01 (Table 1 and Fig. 1b). Excess ergosterol can downregulate the transcription of post-squalene 

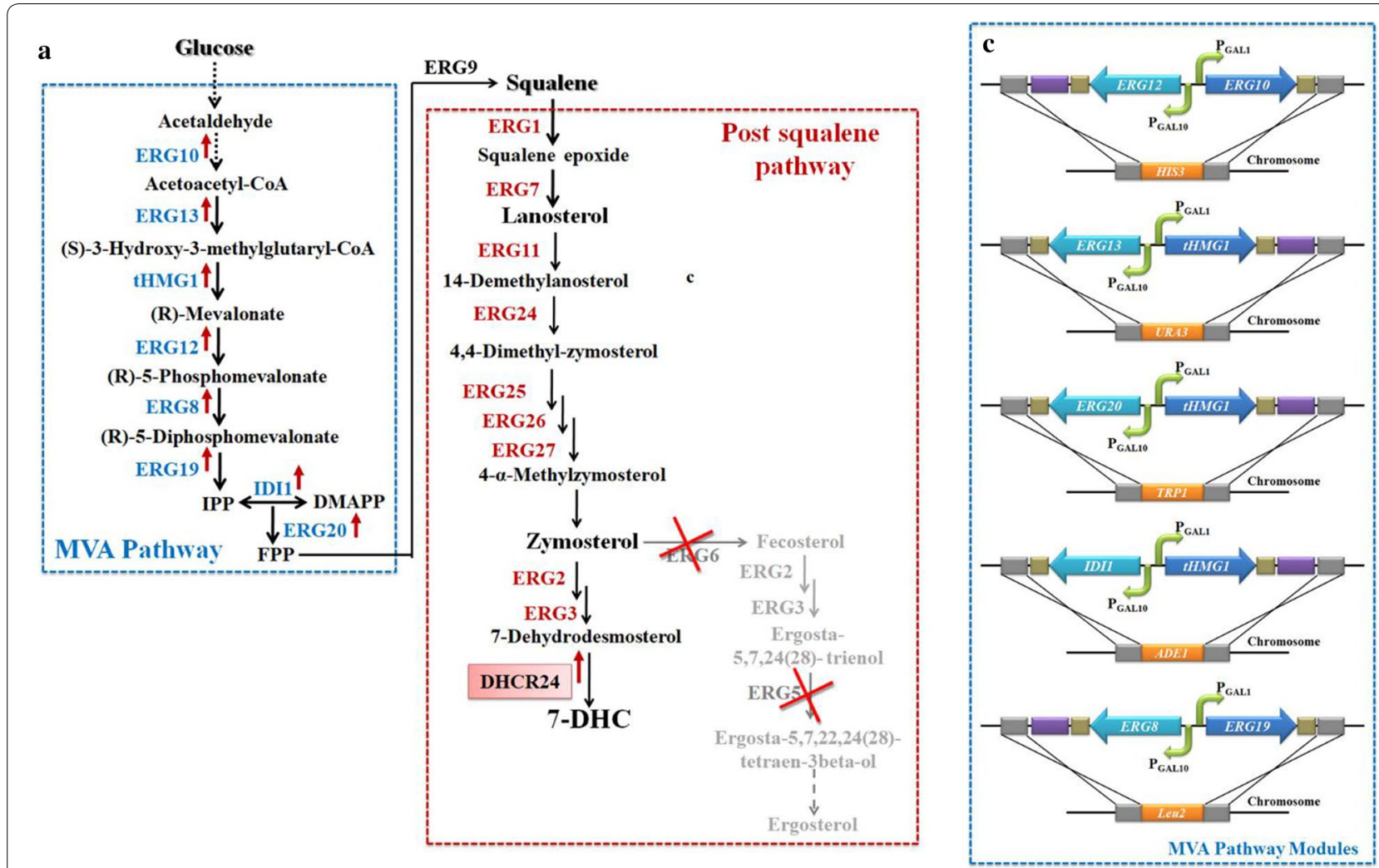

b

7-DHC Overproduction
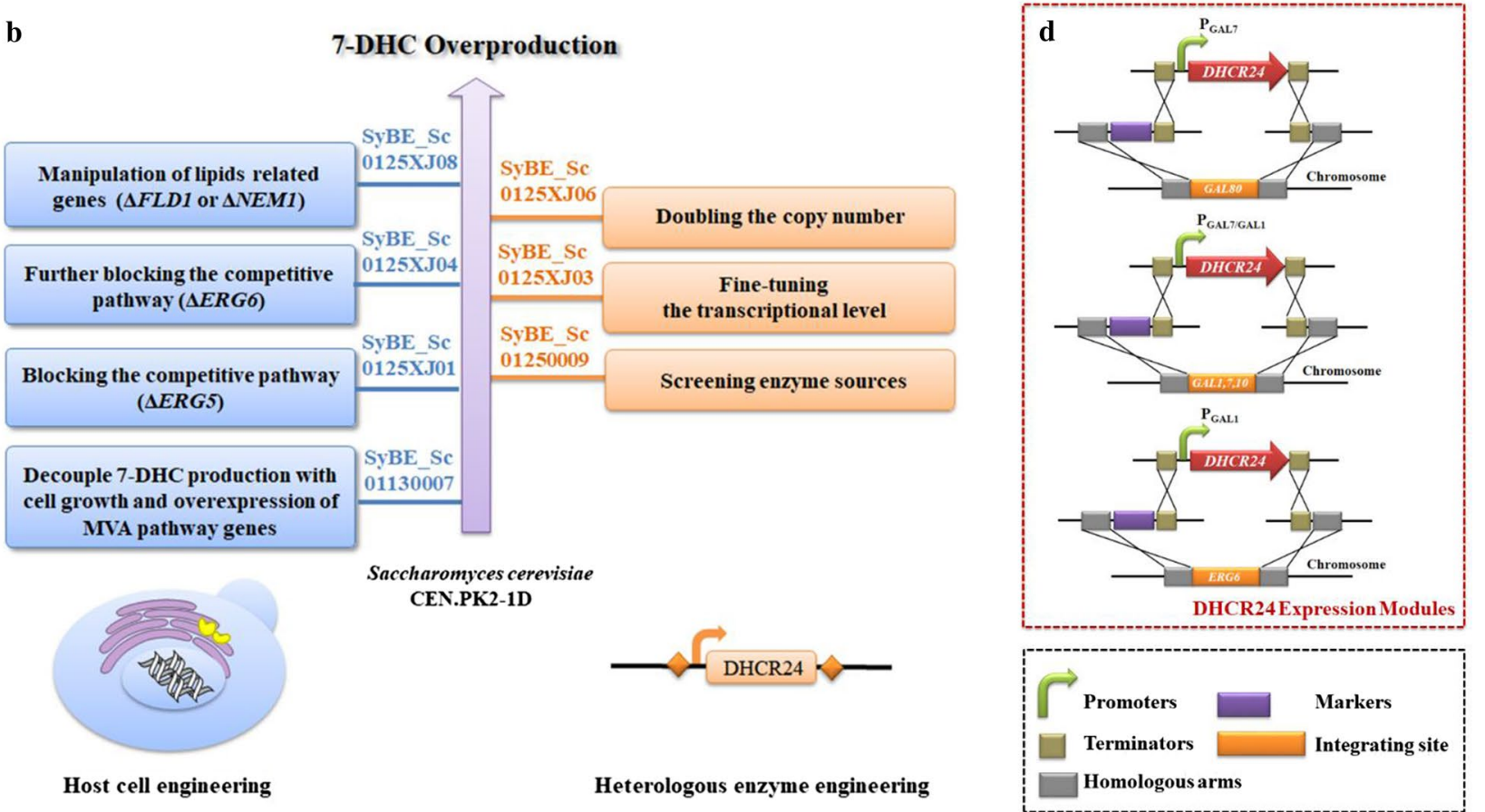

Fig. 1 Overview of 7-DHC biosynthesis pathway and the engineering strategies applied in this study. a Overview of 7-DHC biosynthesis pathway in yeast. The MVA pathway is highlighted in blue and boxed, while the post-squalene pathway is highlighted in red and boxed. The blocked endogenous ergosterol synthesis pathway from zymosterol is illustrated in gray. An upward pointing arrow is used to indicate protein overexpression, and an " $X$ " on a particular enzyme suggests that it is deleted. $\mathbf{b}$ Schematic representation of the engineering strategies to enhance 7-DHC production in S. cerevisiae. The host cell (blue) is engineered in combination with the only heterologous enzyme DHCR24 (orange). c The genetic modification for overexpressing endogenous MVA pathway genes. $\mathbf{d}$ The genetic modification for introducing DHCR24 expression modules along with disruption of GAL80, GAL7,10,1 or ERG6 
Table 1 S. cerevisiae strains used in this study

\begin{tabular}{|c|c|c|}
\hline Strain & Description & Source \\
\hline CEN.PK2-1D & MATa, URA3-52, TRP1-289, LEU2-3112, HIS3 1 1, MAL2-8C, SUC2 & EUROSCARF \\
\hline SyBE_Sc01130007 & 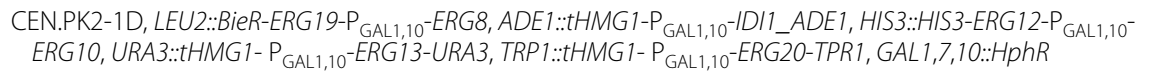 & This study \\
\hline SyBE_Sc0125XJ01 & SyBE_Sc01130007, $\triangle E R G 5$ & This study \\
\hline SyBE_Sc01250050 & SyBE_SC0125XJ01, GAL80::P GAL7 $_{1}-H s \_D H C R 24-T_{\text {PGK1 }}-L E U 2$ & This study \\
\hline SyBE_Sc01250001 & SyBE_Sc0125XJ01, GAL80::P GAL7 -Cg_DHCR24-T PGK1 $-L E U 2$ & This study \\
\hline SyBE_Sc01250002 & SyBE_Sc0125XJ01, GAL80::P GAL7 - Tg_DHCR24-T ${ }_{\text {PGK1 } 1}-L E U 2$ & This study \\
\hline SyBE_Sc01250003 & SyBE_Sc0125XJ01, GAL80::P GAL7 $-M m \_D H C R 24-T_{\text {PGK1 }}-L E U 2$ & This study \\
\hline SyBE_Sc01250004 & SyBE_Sc0125XJ01, GAL80::P GAL7 - At_DHCR24-T ${ }_{\text {PGK1 } 1 \text { LEU2 }}$ & This study \\
\hline SyBE_Sc01250006 & SyBE_SC0125XJ01, GAL80::P GAL7 $-D r \_D H C R 24-T_{\text {PGK1 }}-L E U 2$ & This study \\
\hline SyBE_Sc01250007 & SyBE_Sc0125XJ01, GAL80::P $\mathrm{GAL7}^{-}$Gh_DHCR24-T PGK1-LEU2 & This study \\
\hline SyBE_Sc01250008 & SyBE_SC0125XJ01, GAL80::P GAL7 $_{1}-E C \_D H C R 24-T_{\text {PGK1 }}-L E U 2$ & This study \\
\hline SyBE_Sc01250009 & 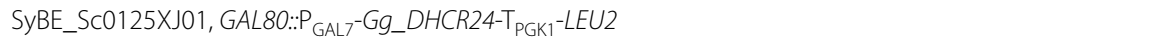 & This study \\
\hline SyBE_Sc01250010 & SyBE_SC0125XJ01, GAL80::P GAL7 $_{1}-X t D D H C R 24-T_{\text {PGK1 }}-L E U 2$ & This study \\
\hline SyBE_Sc01250011 & SyBE_SC0125XJ01, GAL80::P GAL7 $-B t \_D H C R 24-T_{\text {PGK1 }}-L E U 2$ & This study \\
\hline SyBE_Sc0125H001 & SyBE_SC0125XJ01, GAL80::P $\mathrm{GAL}_{7}-\mathrm{Cg}$ DHCR24-6HIS-T ${ }_{\mathrm{PGK} 1}-L E U 2$ & This study \\
\hline SyBE_Sc0125H002 & SyBE_SC0125XJ01, GAL80::P $\mathrm{GAL}-\mathrm{Tg}$ DHCR24-6HIS-T PGK1 -LEU2 & This study \\
\hline SyBE_Sc0125H003 & SyBE_Sc0125XJ01, GAL80::P GAL7 $-M m$ DHCR24-6HIS-T ${ }_{\text {PGK1 }}-L E U 2$ & This study \\
\hline SyBE_Sc0125H005 & SyBE_Sc0125XJ01, GAL80::P $\mathrm{PAL}_{\text {GL-At DHCR24-6HIS-T PGK1 }}$-LEU2 & This study \\
\hline SyBE_Sc0125H007 & SyBE_SC0125XJ01, GAL80::P GAL7-DrDHCR24-6HIS-T $_{\text {PGK1 }}-$ LEU2 & This study \\
\hline SyBE_Sc0125H009 & 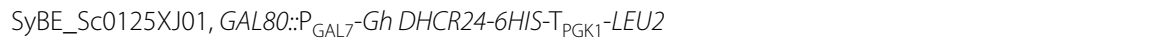 & This study \\
\hline SyBE_Sc0125H010 & SyBE_SC0125XJ01, GAL80::P $\mathrm{PAL}_{\text {GL }}-E C$ DHCR24-6HIS-T PGK1 $-L E U 2$ & This study \\
\hline SyBE_Sc0125H011 & SyBE_SC0125XJ01, GAL80::P $\mathrm{PAL}_{7}-\mathrm{Gg}$ DHCR24-6HIS-T $\mathrm{PGK1}-L E U 2$ & This study \\
\hline SyBE_Sc0125H012 & SyBE_SC0125XJ01, GAL80:: GAL7 $-X t$ DHCR24-6HIS-T PGK1 $^{-L E U 2}$ & This study \\
\hline SyBE_Sc0125H013 & SyBE_SC0125XJ01, GAL80::P GAL7 $^{-B t}$ DHCR24-6HIS-T PGK1 $^{-L E U 2}$ & This study \\
\hline SyBE_Sc0125H050 & SyBE_SC0125XJ01, GAL80::P GAL7 $_{1}-$ Hs DHCR24-6HIS-T ${ }_{\text {PGK1 }}-L E U 2$ & This study \\
\hline SyBE_Sc0125XJ02 & SyBE_SC0125XJ01, GAL7,10,1::P GAL7-Gg_DHCR24-T $\mathrm{CYC1}_{1}-U R A 3$ & This study \\
\hline SyBE_Sc0125XJ03 & SyBE_SC0125XJ01, GAL7,10,1::P $\mathrm{PAL1}_{1}-\mathrm{Gg}_{-} D H C R 24-\mathrm{T}_{\mathrm{CYC} 1}-U R A 3$ & This study \\
\hline SyBE_Sc0125X001 & 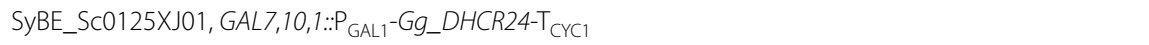 & This study \\
\hline SyBE_Sc0125XJ04 & SyBE_Sc0125XJ01, GAL7,10,1::P GAL1 $-G_{-}$DHCR24-T CYC1, ERG6::LEU2 & This study \\
\hline SyBE_Sc0125XJ06 & 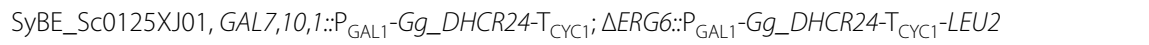 & This study \\
\hline SyBE_Sc0125XJ07 & SyBE_SC0125XJ06, FLD1::URA3 & This study \\
\hline SyBE_Sc0125XJ08 & SyBE_Sc0125XJ06, NEM1::URA3 & This study \\
\hline SyBE_Sc0125XJ09 & SyBE_Sc0125XJ06, FLD1::KanMX, NEM1::URA3 & This study \\
\hline
\end{tabular}

genes, and it was presumed that ergosterol defect would trigger sterol feedback system (such as ECM22/UPC2) $[19,20]$ to upregulate the genes in $7-\mathrm{DHC}$ synthesis pathway [7]. Here, transcriptional analysis of strain SyBE_ Sc01130007 (control) and SyBE_Sc0125XJ01 (DERG5) revealed that disruption of ERG5 significantly activated the transcription of all of the MVA genes (Additional file 1: Figure $\mathrm{S} 1 \mathrm{a}-\mathrm{h}$ ) and majority of the post-squalene genes (except ERG24, ERG27, and ERG6, Additional file 1: Figure S1i-r) during ethanol consumption phase. The transcriptional levels of genes ERG13, tHMG1, ERG20, ERG11, ERG25, and ERG3 were even enhanced during glucose consumption phase (Additional file 1:
Figure S1). Notably, the transcriptions of MVA pathway genes were jointly controlled by their native promoters and GAL promoters. The upregulation efforts on these promoters were mainly represented when glucose was exhausted (Additional file 1: Figure S1), indicating a potential cross talk between galactose regulon and sterol homoeostasis. Thus, deletion of the endogenous gene ERG5 was beneficial to 7-DHC production not only in terms of blocking the metabolic bypass but also via abolishing the suppressive effect of ergosterol on sterol synthesis pathway. And the improvement on the activities of GAL promoters by $\triangle E R G 5$ would be beneficial for the expression of heterologous genes. Eventually, introducing 
DHCR24 from Homo sapiens (Hs_DHCR24) [21] generated $36.1 \mathrm{mg} / \mathrm{L}$ 7-DHC in the host SyBE_Sc0125XJ01 (Fig. 2).

\section{Screening DHCR24 sources}

As proved in many cases, screening enzymes from diverse sources is a promising strategy to enhance the titer of desired product [22-24]. In this study, DHCR24 is the only heterologous protein which catalyzed the final step in 7-DHC synthesis pathway (Fig. 1a). So far, only three DHCR24s, which were from $H$. sapiens, M. musculus (Mm_DHCR24) [25], and Danio rerio (Dr_DHCR24) [26], have been adopted to synthesize 7-DHC [5, 6]. However, their activities have not been compared. In this study, except these three DHCR24s, four vertebrate DHCR24s from Equus caballus (Ec_DHCR24) [27], Gallus gallus (Gg_DHCR24) [28], Xenopus tropicalis (Xt_DHCR24) [29], and Bos taurus (Bt_DHCR24) [30]; two plant DHCR24s from Arabidopsis thaliana (At_DHCR24) [31] and Gossypium hirsutum (Gh_DHCR24); one invertebrate DHCR24 from Trypanosoma grayi (Tg_DHCR24) [32]; and one fungal DHCR24 from Cryptococcus gattii (Cg_DHCR24) [33] were selected (Fig. 2a and Table 2) and introduced into strain SyBE_Sc0125XJ01. As illustrated in Additional file 1: Figure S2, all the strains carrying different DHCR24s presented comparable cell growths in YPD medium. Meanwhile, none of DHCR24s from plant, invertebrate, or fungus has realized 7-DHC accumulation in yeast (Fig. 2b), even though these DHCR24s were successfully expressed in hosts (Fig. 2c). As reported, DHCR24 homologs in plants first catalyze the isomerization of the $\Delta^{24(28)}$ bond, and then deoxidize the $\Delta^{24(25)}$ bond in sterol substrate [30]. Therefore, it was speculated that plant DHCR24s require an isomeric substrate rather than 7-dehydrodesmosterol to realize the desired $\Delta^{24}$-reduction step for 7-DHC synthesis. In contrast, vertebrate DHCR24s, activities of which do not cover the initial isomerization reaction, could achieve 7-DHC synthesis in yeast at different levels (Fig. 2b). Among the seven tested vertebrate proteins, Gg_DHCR24 obtained the highest 7 -DHC production $(64.1 \mathrm{mg} / \mathrm{L}$, Fig. $2 \mathrm{~b})$ which was 1.8 -fold of that realized by $H s_{-}$DHCR24. And western-blotting assay revealed there was no statistic difference in the expression levels of DHCR24s among different vertebrate species except for $M m_{-}$DHCR24 (which achieved higher expression level) (Fig. 2c), suggesting $G g \_$DHCR24 might process higher enzyme activity for $\Delta^{24}$-reduction. Thus, Gg_DHCR24 was selected for next-step construction of 7-DHC overproducing strain. And improvements on the expression of this enzyme are probably needed to boost 7-DHC titer further.

\section{Enhancing the transcriptional level of DHCR24 via modifying its induction strategy, integration position, and used promoter}

To employ GAL promoters, GAL7,1,10 were knocked out to eliminate galactose utilization [34]. And initially, $\triangle G A L 80$ was applied to avoid addition of the inducer [34]. As is well known, there is another routine strategy for galactose-regulation, i.e., only deleting $G A L 7,1,10$ and leaving GAL80 untouched [17]. In this atudy, both these strategies were tested on the transcription levels of DHCR24 and even 7-DHC production. In brief, in the control strain SyBE_Sc01250009, DHCR24 expression cassette $\left(\mathrm{P}_{\mathrm{GAL} 7}-D H C R 24\right)$ was integrated into locus GAL80; whereas in strain SyBE_Sc0125XJ02, the same DHCR24 expression cassette was inserted into locus $G A L 7,1,10$, leaving a wild-type $G A L 80$ (Table 1). As illustrated in Fig. 3a, before galactose induction (glucose consumption phase), the basic transcription level of DHCR24 is reduced by $94.5 \%$ in strain SyBE_Sc0125XJ02 than that in the control strain SyBE_Sc01250009, suggesting galactose regulation is stricter under wild-type GAL80 than that under $\triangle G A L 80$. Correspondingly, weaker promoter leakage of DHCR24 during glucose consumption phase would improve biomass build-up by alleviating cell toxicity brought forth by 7-DHC synthesis, which might be supported by the better cell growth of GAL80 wild-type strain (SyBE_Sc0125XJ02 and SyBE_Sc0125XJ03) than that of $\triangle G A L 80$ strain (SyBE_Sc01250009) (Additional file 1: Figure S3). In the meanwhile, after being activated by galactose, the transcription level of DHCR24 in strain SyBE_Sc0125XJ02 is $26.8 \%$ higher than that in the control strain (Fig. 3a). Consequently, the 7-DHC production was enhanced by $19.6 \%$ (to $78.4 \mathrm{mg} / \mathrm{L}$ ) through altering

\footnotetext{
(See figure on next page.)

Fig. 2 Effect of enzyme sources of DHCR24 on 7-DHC production. a Phylogenetic analysis of DHCR24 protein sequences selected in this study. $\mathbf{b}$ 7-DHC production in strains with DHCR24s from diversity species. Those DHCR24s that could not realize 7-DHC accumulation are denoted by red triangle. $\mathbf{c}$ Western-blotting of lysates from cells expressing polyhistidine-tag-attached DHCR24s from the selected sources. Cells were cultured in YPD medium and harvested at $40 \mathrm{~h}$ (ethanol consumption phase). Extracts were probed with anti-polyhistidine and anti-GAPDH (as loading control). The relative expression level of each DHCR24 is displayed as the gray scale of anti-polyhistidine band divided by that of anti-GAPDH. The error bars represent standard deviation calculated from triplicate experiments. Hs, Homo sapiens; Mm, Mus musculus; Dr, Danio rerio; Ec, Equus caballus; Gg, Gallus gallus; Xt, Xenopus tropicalis; Bt, Bos Taurus; At, Arabidopsis thaliana; Gh, Gossypium hirsutum; Cg, Cryptococcus gattii; Tg, Trypanosoma grayi
} 

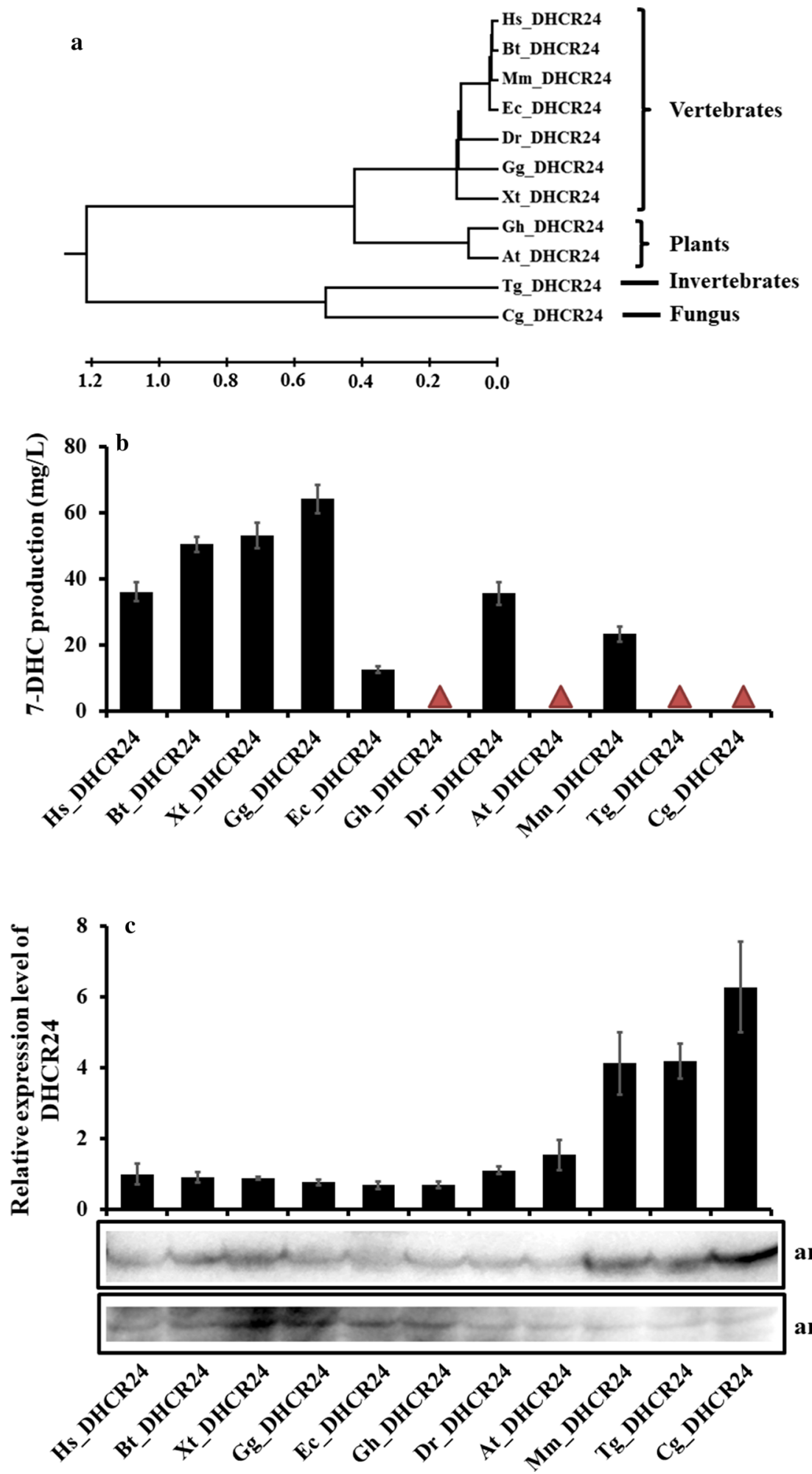

anti-polyHistidine anti-GAPDH 
Table 2 DHCR24s employed in this study

\begin{tabular}{|c|c|c|c|c|}
\hline Protein & Species & Accession no. & Reaction & References \\
\hline \multicolumn{5}{|c|}{ Vertebrate DHCR24s } \\
\hline HS_DHCR24 & Homo sapiens & Q15392 & Catalyzing $\Delta^{24}$-reduction of sterol substrate & {$[21]$} \\
\hline Mm_DHCR24 & Mus musculus & Q8VCH6.1 & Catalyzing $\Delta^{24}$-reduction of sterol substrate & {$[25]$} \\
\hline Dr_DHCR24 & Danio rerio & AAl65211.1 & Catalyzing $\Delta^{24}$-reduction of sterol substrate & {$[26]$} \\
\hline EC_DHCR24 & Equus caballus & NP_001157423.1 & N.P & {$[27]$} \\
\hline Gg_DHCR24 & Gallus gallus & NP_001026459.1 & N.P & {$[28]$} \\
\hline Xt_DHCR24 & Xenopus tropicalis & NP_001016800.1 & N.P & [29] \\
\hline Bt_DHCR24 & Bostaurus & AAl50074.1 & N.P & {$[30]$} \\
\hline \multicolumn{5}{|l|}{ Plant DHCR24s } \\
\hline At_DHCR24 & Arabidopsis thaliana & Q39085.2 & $\begin{array}{l}\text { Catalyzing } \Delta^{24(28)} \text {-isomerization first, and then } \Delta^{24} \text { - } \\
\text { reduction of sterol substrate }\end{array}$ & {$[31]$} \\
\hline Gh_DHCR24 & Gossypium hirsutum & NP_001314012 & N.P & N.P \\
\hline \multicolumn{5}{|c|}{ Invertebrate DHCR24 } \\
\hline Tg_DHCR24 & Trypanosoma grayi & XP_009306481.1 & N.P & {$[32]$} \\
\hline \multicolumn{5}{|l|}{ Fungal DHCR24 } \\
\hline Cg_DHCR24 & Cryptococcus gattii & XP_003192961.1 & N.P & [33] \\
\hline
\end{tabular}

N.P not published
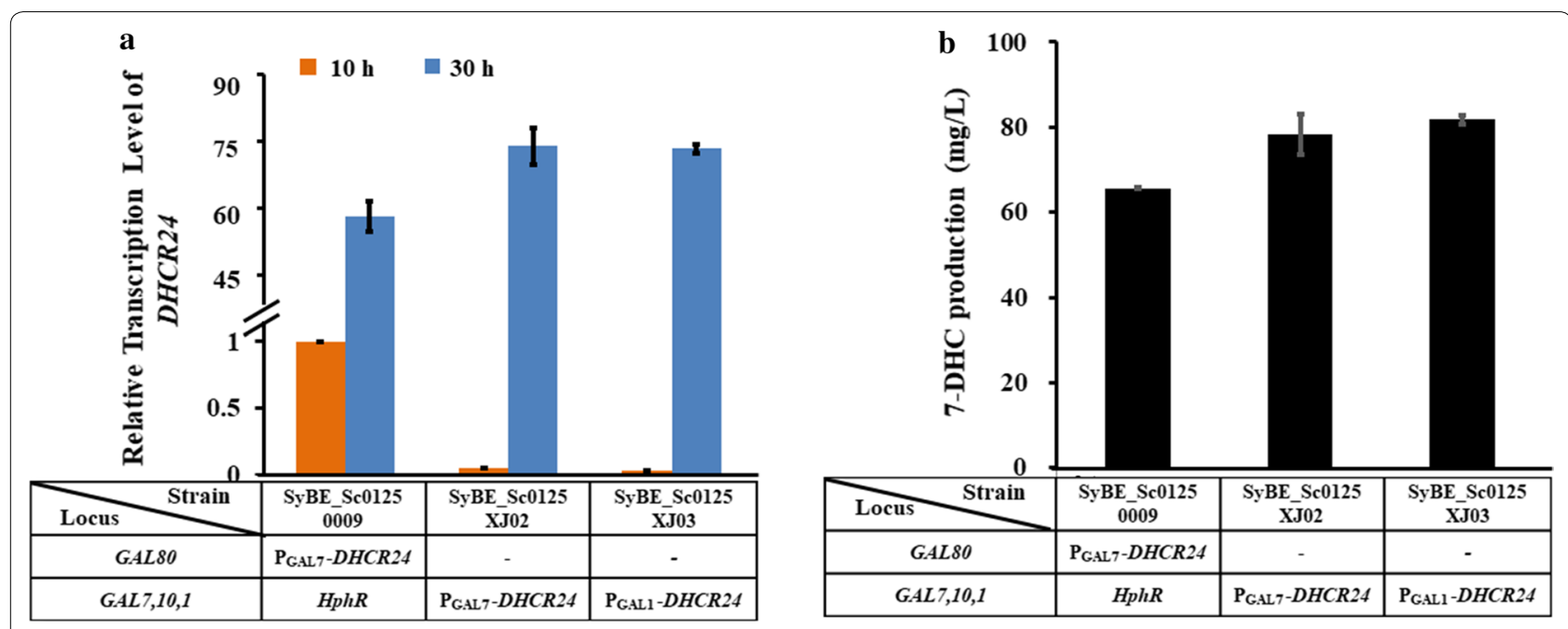

Fig. 3 Fine-tuning the transcription level of DHCR24 to enhance 7-DHC production. a Real-Time PCR analysis of the transcription levels of DHCR24 in strains harvested at $10 \mathrm{~h}$ (orange, glucose consumption phase) and $30 \mathrm{~h}$ (blue, ethanol consumption phase). The relative transcription level for each gene was determined as $2^{-\Delta \Delta C t}$ using gene ALG9 for normalization. All data were from at least triplicate experiments. $\mathbf{b}$ Effects of the induction strategy, genomic integration site, and promoter of DHCR24 on 7-DHC accumulation

the induction strategy (Fig. 3b). Besides that, it is hard to ignore the integration position effects on gene expression in S. cerevisiae [35]; thus, the improvement on the transcription level of DHCR24 as well as the increase on 7 -DHC production is also brought forth by the changes on the integration position of DHCR24 expression cassette within yeast genome.

Initially, the expressions of DHCR24 were controlled by promoter GAL7 $\left(\mathrm{P}_{\mathrm{GAL7}}\right)$. As it was reported that the activity of promoter GAL1 $\left(\mathrm{P}_{\mathrm{GAL} 1}\right)$ was stronger than that of $\mathrm{P}_{\text {GAL7 }}$ [34], the promoter of DHCR24 in strain SyBE_Sc0125XJ02 was replaced by $\mathrm{P}_{\mathrm{GAL} 1}$, generating strain SyBE_Sc0125XJ03 (Table 1). However, the transcription level of DHCR24 was not increased correspondingly (Fig. 3a), indicating that promoter activity might be affected by the particular host environment. As a result, little improvement on 7-DHC (from 78.4 to $81.7 \mathrm{mg} / \mathrm{L}$ ) was detected by comparing that in strain 
SyBE_Sc0125XJ03 with strain SyBE_Sc0125XJ02 (Fig. 3b). Despite this, since strain SyBE_Sc0125XJ03 achieve higher 7-DHC titer, this strain was still chosen for the next step of optimization. Worthy to be noticed, there was positive association between 7-DHC accumulation and DHCR24 transcriptional level (Fig. 3). Thus, further improving the transcription level of DHCR24, such as doubling its copy number, might be a promising approach to enhance 7-DHC production.

\section{Further blocking sterol competitive pathway by $\triangle E R G 6$}

In our current sterol biosynthesis pathway, there was still ERG6 existing to convert zymosterol to fecosterol, which is not required for 7-DHC synthesis. To block this by-path, gene ERG6 was knocked out in strain SyBE_Sc0125XJ03 by marker LEU2, gaining strain SyBE_Sc0125XJ04 (Table 1 and Fig. 1b). As a result, deletion of ERG6 enhanced the accumulation of its substrate zymosterol (Fig. 4a, c). Besides that, this approach also reduced the accumulation of squalene (Fig. $4 \mathrm{~b}$ ) as well as increasing the accumulation of lanosterol (Fig. 4d), indicating enlargement of the metabolic flow through post-squalene pathway. Consequently, 7-DHC production was increased by $77.6 \%$ (to $145.1 \mathrm{mg} / \mathrm{L}$ ) by $\triangle E R G 6$ (Fig. 4e). Then, adding another copy of DHCR24 expression cassette $\left(\mathrm{P}_{\mathrm{GAL1}}-\right.$ DHCR24) into locus ERG6 (obtaining strain SyBE_Sc0125XJ06, Table 1 and Fig. 1b) further improved 7-DHC titer by $16.5 \%$ (to $169.1 \mathrm{mg} / \mathrm{L}$, Fig. 4e). Strains SyBE_Sc0125XJ03, SyBE_Sc0125XJ04, and SyBE_Sc0125XJ06 demonstrated comparable cell growths in YPD medium (Additional file 1: Figure S4). Therefore, strain SyBE_Sc0125XJ06 was employed for further engineering.

\section{Engineering lipids metabolism genes}

As described above, endogenous sterol accumulation was enhanced by $\triangle F L D 1$ [10] as well as by $\triangle P A H 1$ [11]. Meanwhile, NEM1 (YHR004C) is the catalytic subunit of NEM1-SPO7 phosphatase, which is responsible for dephosphorylation of PAH1 to activate its function [36]. Therefore, besides deletion of FLD1, knocking out NEM1 would also be benefit for 7-DHC production. Accordingly, these two genes were individually knocked out in strain SyBE_Sc0125XJ06, generating strains SyBE_Sc0125XJ07 ( $\triangle F L D 1)$ and SyBE_Sc0125XJ08 ( $\triangle N E M 1)$, respectively (Table 1). As illustrated in Fig. 5a, using strain SyBE_Sc0125XJ06 as the control, $\triangle F L D 1$ and $\triangle N E M 1$ achieved $15.7 \%$ (to $195.7 \mathrm{mg} / \mathrm{L}$ ) and $48.3 \%$ (to $250.8 \mathrm{mg} / \mathrm{L}$ ) improvement on 7-DHC production, respectively. However, further combination of $\triangle F L D 1 /$ $\triangle N E M$ reduced the $7-\mathrm{DHC}$ titer to $109.0 \mathrm{mg} / \mathrm{L}$ (Fig. $5 \mathrm{a}$ ). Therefore, NEM1-deleted strain SyBE_Sc0125XJ08 was employed in further fed-batch fermentation. Besides, 7-DHC titer of strain SyBE_Sc0125XJ08 in YPD medium was 8.25 -fold higher than that achieved in SC medium with the same glucose concentration, while the biomass $\left(\mathrm{OD}_{600}\right)$ under YPD medium when harvested was only 1.25 -fold higher than that under SC medium (Additional

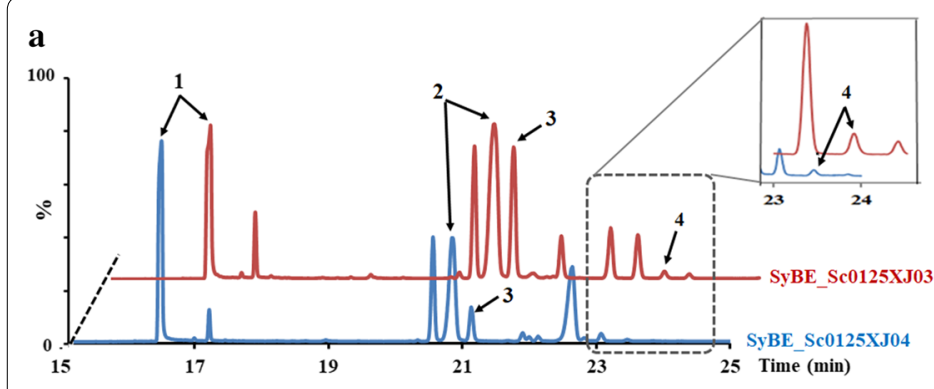

b
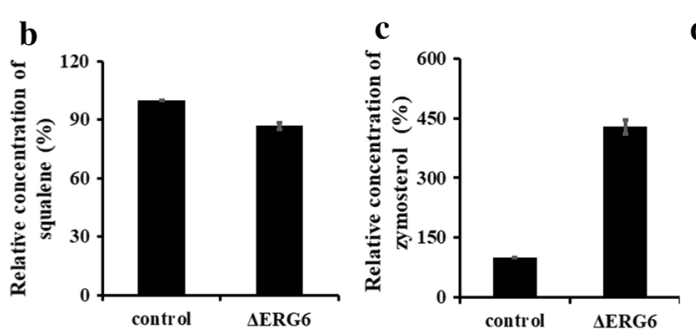

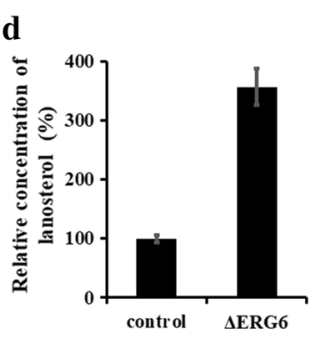

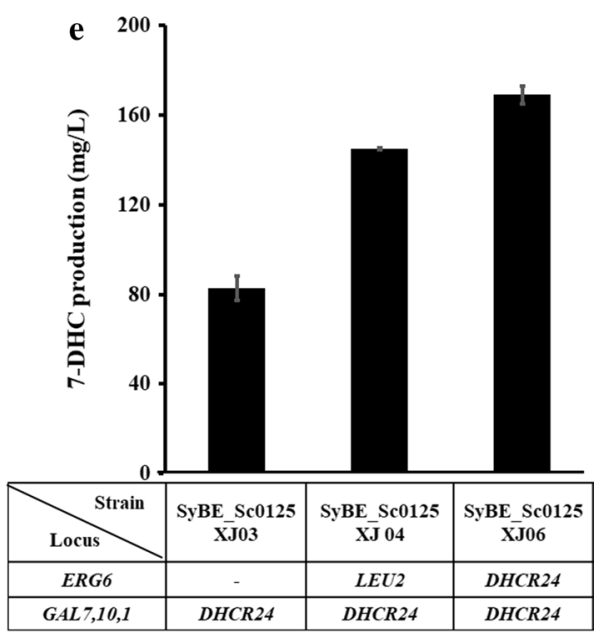

Fig. 4 Effect of $\triangle E R G 6$ on 7-DHC production. a GC/TOF-MS analysis of the fermentation products of strains SyBE_SC0125XJ03 (red) and SyBE_ Sc0125XJ04 (blue). I, squalene; II, 7-DHC; III, zymosterol; IV, lanosterol. The relative accumulations of squalene (b) zymosterol (c), and lanosterol (d) in strains SyBE_Sc0125XJ03 (control) and SyBE_Sc0125XJ04 ( $\triangle E R G 6$ ) were determined using those in the control strain for normalization. e Improvement of 7-DHC accumulation via blocking the competitive path ( $\triangle E R G 6$ ) and supplementing another copy of DHCR24 in locus ERG6. The error bars represent standard deviation calculated from triplicate experiments 


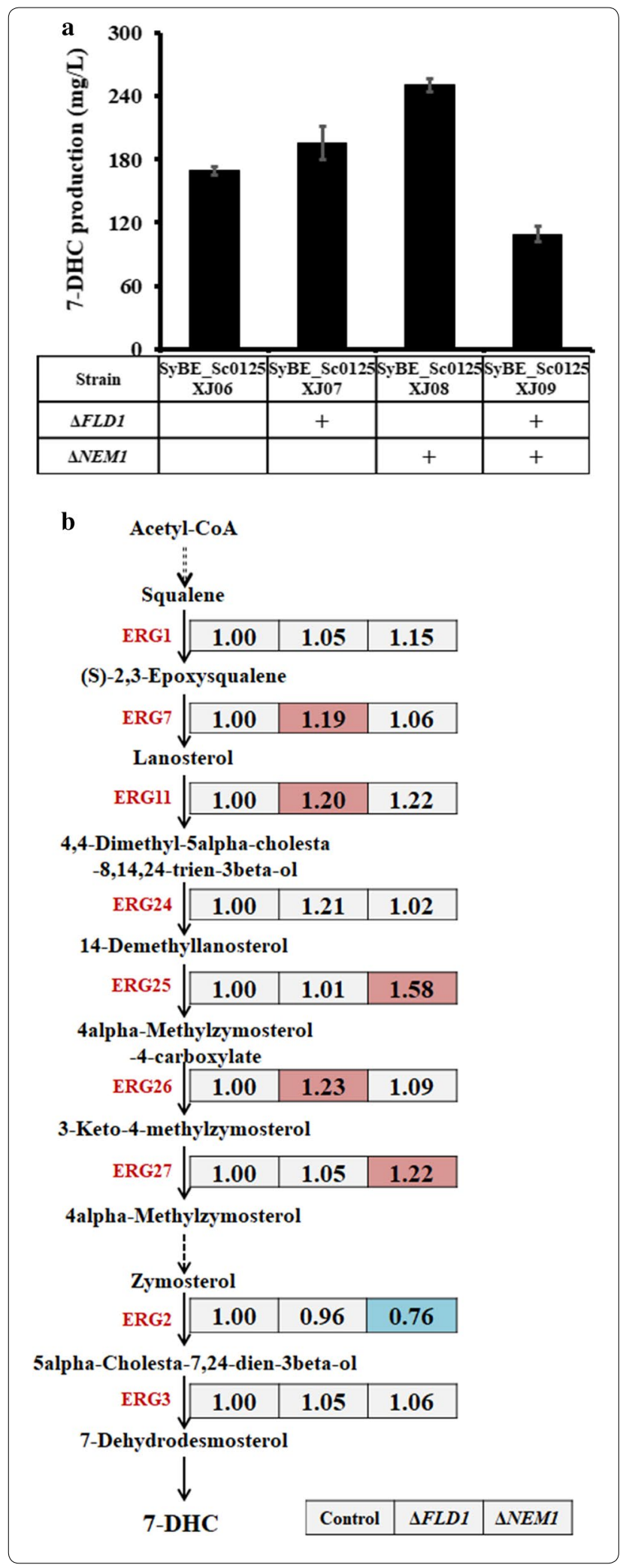

Fig. 5 Effect of deleting lipids metabolism associated genes on 7-DHC production. a 7-DHC production in the control (SyBE_ Sc0125XJ06) and strains with individual deletion of FLD1 and NEM1. b Relative transcription levels of the post-squalene pathway genes in strains SyBE_Sc0125XJ06 (control), SyBE_Sc0125XJ07 ( $\triangle F L D 1$ ), and SyBE_SC0125XJ08 ( $\triangle N E M 1$ ). Cells were harvested at $30 \mathrm{~h}$ (ethanol consumption phase). The relative transcription level for each gene was quantified by Real-Time PCR and determined as $2^{-\Delta \Delta C t}$ using gene ALG9 for normalization (data listed in the box). All data were from at least triplicate experiments. Significance levels of $t$ test were determined for $P<0.05$. Upregulated, downregulated genes, and genes without significant transcriptional difference are denoted in red, blue, and gray, respectively

file 1: Figure S5). These data demonstrated the unknown effect of complex media on the 7-DHC titers besides boosting cell growth.

Deletion of $\triangle F L D 1$ or $\triangle N E M 1$ both demonstrated modification on the profile of cellular lipids, including triacylglycerols, sterols and phospholipids, which are all compositions of cell membrane [10,11]. Altering membrane lipid composition is a crucial for microbial stress adaptation [37]. However, neither deletion of FLD1 nor NEM1 could improve cell growth of 7-DHC synthesis strain in YPD medium (Additional file 1: Figure S5a), suggesting their positive effect on 7-DHC output might not due to improvement on bacterial stress adaptation by altering membrane compositions. Further, the effect of losing FLD1 or $\triangle N E M 1$ upon 7-DHC synthesis was investigated on transcriptional level. As shown in Fig. 5b, deletion of $F L D 1$ significantly upregulated post-squalene genes ERG7, ERG11, and ERG26. Overexpression of ERG11 resulted in increase of downstream sterols (such as 4,4-dimethylzymosterol, zymosterol and ergosterol) in ergosterol synthetic yeast [38], which might be the reason for the increased 7-DHC productivity by $\triangle F L D 1$. Meanwhile, as reported by Arendt et al. [39], $\triangle P A H 1$ stimulated a dramatic expansion of the endoplasmic reticulum (ER), which resulted in overproduction of triterpenoids (e.g., $\beta$-amyrin and its derivatives) probably by functional overproduction of ER-localized proteins. However, among those ER-located genes (ERG1, ERG11, ERG24-27, and ERG2-3) [40], only ERG25 and ERG27 was significantly activated by $\triangle N E M 1$, indicating that the enhanced 7-DHC productivity by $\triangle N E M 1$ might not bring by ER-engineering effect. Thus, it is probably required further global transcription analysis by RNAsequencing to expose its functional mechanism toward 7-DHC synthesis. 


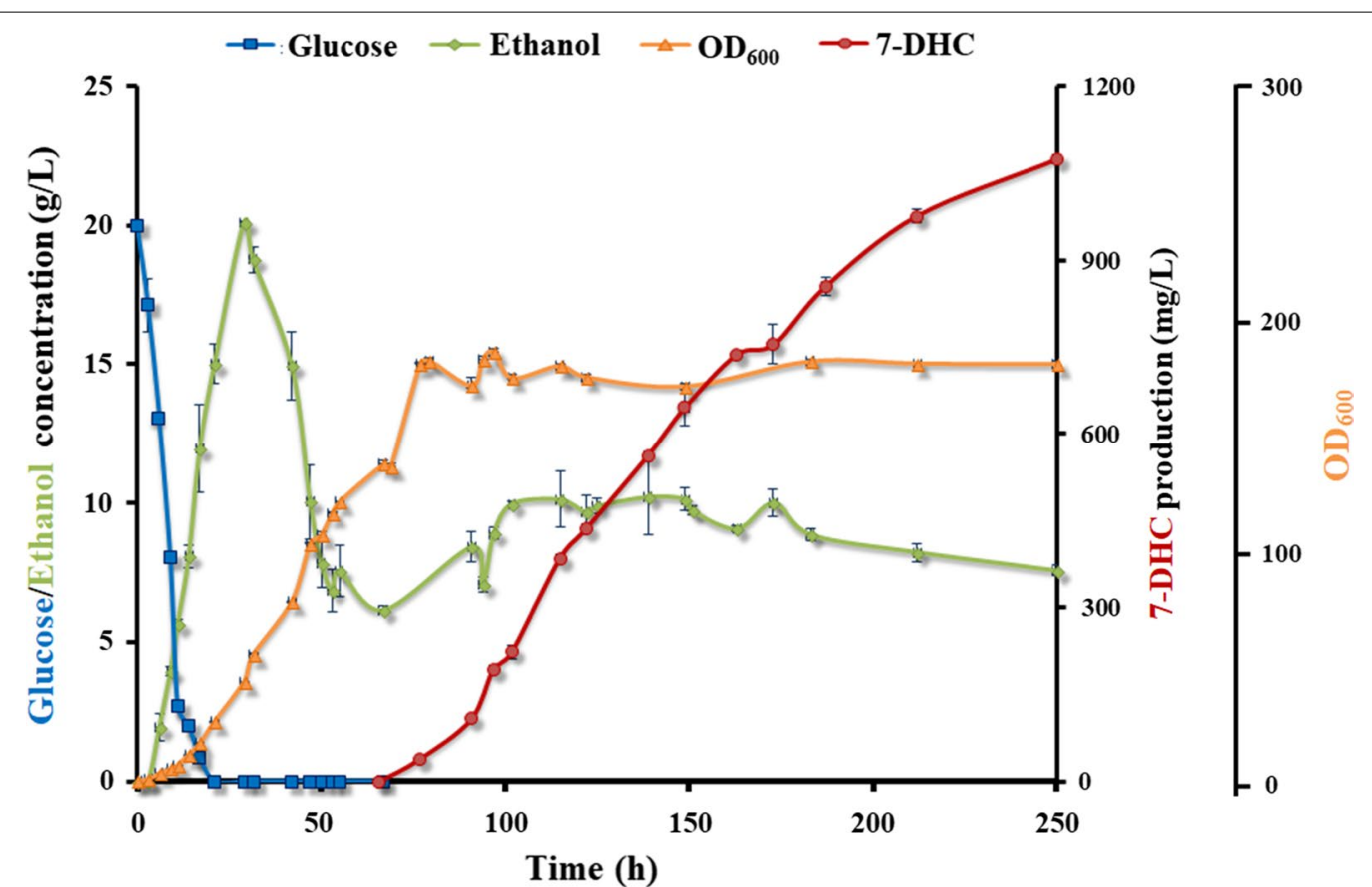

Fig. 6 7-DHC production in fed-batch fermentation. Profile 7-DHC titer (red), glucose (blue), ethanol (green), and biomass (orange) during fermentation with strain SyBE_Sc0125XJ08. The error bars represent standard deviation calculated from duplicate experiments

\section{Fed-batch fermentation}

Fed-batch fermentation of strain SyBE_Sc0125XJ08 was carried out in 5-L bioreactor under glucose restriction strategy. Glucose concentration was controlled below $5 \mathrm{~g} / \mathrm{L}$ for reducing the ethanol and glycerol produced during the fermentation process (Fig. 6). Air flow and dissolved oxygen were controlled appropriately as sterols synthesis in S. cerevisiae was an oxygen consumption process and too much oxygen supply would inhibit sterol synthesis [41]. As the usage of GAL promoters to control 7-DHC synthesis, the whole process was divided into cell growth stage and 7-DHC accumulation stage. In the first stage, glucose was used as the solo carbon source for biomass growth. In the second stage, galactose was added into the bioreactors, and glucose was not further supplied. During that time, ethanol was the carbon source mainly for 7-DHC accumulation. Eventually, after 250-h cultivation, 7-DHC production reached $1.07 \mathrm{~g} / \mathrm{L}$ (Fig. 6), which is the highest reported microbial titer as yet known.

However, 7-DHC synthesis could be further enhanced via host engineering in yeast. On the one hand, ergosterol is essential to maintain a normal structure and function of cellular membranes [42], and ergosterol defect could also trigger redox imbalance [7]. Therefore, besides ergosterol supplement, introducing cofactor regeneration modules and building gene genetic to restrict transcription of ERG5-6 only in cell growth stage would compensate for the necessary block of ergosterol biosynthesis during 7-DHC accumulation period. One the other hand, sterols stored in S. cerevisiae in their esterified forms, and overexpression of two endogenous sterol acyltransferases (ARE1 and ARE2) could promotes sterols accumulation $[43,44]$. However, a DSM patent revealed that reducing or abolishing the activity of ARE1 or ARE2 was beneficial to 7-DHC production in yeast [45]. Even though it is hard to explain the contrary results of these works, these data suggested that modifying formation and hydrolysis of sterol esters would be another promising approach to boost 7-DHC output in future study.

\section{Conclusions}

In this work, combined engineering of the host cell and the heterologous enzyme DHCR24 significantly improved 7-DHC productivity in S. cerevisiae. A modified host cell was constructed to appeal to the increased 7-DHC accumulation via decoupling 7-DHC production with cell growth, enhancing MVA pools, totally blocking the competitive path $(\triangle E R G 5,6)$, as well as deleting lipids metabolism gene $(\triangle N E M 1)$. In the meanwhile, the optimal DHCR24 sources (Gg_DHCR24) were obtained by screening the enzymes from diversity species. And through fine-tuning the transcription level of Gg_DHCR24 in terms of adjusting its induction strategy $(\triangle G A L 7,1,10)$, integration position (loci $G A L 7,1,10$, and ERG6), used promoter type $\left(\mathrm{P}_{\mathrm{GAL} 1}\right)$, and copy numbers, 7 -DHC production were stepwise improved accordingly. 
Eventually, the highest 7-DHC titer, so far known $(1.01 \mathrm{~g} / \mathrm{L})$, was achieved in 5 - $\mathrm{L}$ bioreactor, which is 26.9 fold higher than that of the starting strain. This work not only opens large opportunities to realizes downstream the de novo synthesis of other steroids, but also highlights the importance of the combinatorial engineering of heterologous pathway and host to obtain microbial overproduction of many other natural products.

\section{Methods}

\section{Strains and media}

Escherichia coli $\mathrm{DH} 5 \alpha$, which was used for plasmids construction, was cultivated at $37{ }^{\circ} \mathrm{C}$ in Luria-Bertani (LB) medium supplemented with $50 \mu \mathrm{g} / \mathrm{mL}$ kanamycin. The yeast strains used in this study were derived from S. cerevisiae CEN.PK2-1D and summarized in Table 1. Recombinational yeasts were selected on solid synthetic complete (SC) medium lacking appropriate nutrient component [46]. Shake flask fermentation of engineered strains was performed in modified YPD medium (2\% peptone, $1 \%$ yeast extract, $4 \%$ glucose and $1 \% \mathrm{D}-(+)-$ galactose) at $30^{\circ} \mathrm{C}$.

\section{Protein analysis}

The protein sequences of the selected DHCR24s from $H$. sapiens, M. musculus, D. rerio, E. caballus, G. gallus, X. tropicalis, B. taurus, A. thaliana, G. hirsutum, C. gattii, T. grayi were obtained from NCBI database (https://www. ncbi.nlm.nih.gov/, Table 2). Protein sequences alignment and phylogenetic tree construction were carried out with MEGA7 [47].

\section{Construction of plasmids and strains}

Yeast homologous recombination was applied to knockout genes as well as to integrate genes expression cassettes. All the primers used in this study were synthesized by Genewiz Inc. (China) and listed in Additional file 1: Table S1. All the auxotroph markers, promoters, and terminators adopted here were obtained from our module library SynbioML (http://synbioml.org/). Heterologous DHCR24 genes were codon-optimized (Additional file 1: Table S2) and synthesized by GenScript Inc. (China). All the endogenous genes involved in this study were PCR amplified from the genomic DNA of $S$. cerevisiae CEN. PK2-1D. These PCR products shared 40-bp ends homologous to the adjacent fragments or linearized vector; therefore, MVA pathway-enhancing cassettes (Fig. 1c) can be constructed by Gibson assembly method [48]. In the meanwhile, homologous arm cassettes and DHCR24 expression cassettes (Fig. 1d) were assembled by overlap extension PCR (OE-PCR). The assembled products were cloned into plasmid pRS425K (Additional file 1:
Table S3). Before yeast transformation via the LiAc/SS carrier DNA/PEG method [46], these plasmids should be treated by enzyme(s) digestion.

\section{Shake-flask and fed-batch fermentation}

For shake flask fermentation, glycerol-stock yeasts were rejuvenated on solid YPD plate [46]. Then a single colony was picked up and inoculated into $5 \mathrm{~mL}$ YPD medium for overnight cultivation at $30{ }^{\circ} \mathrm{C}$. When cells entered mid-exponential phase, the culture was transferred into $50 \mathrm{~mL}$ modified YPD medium or SC medium with an initial $\mathrm{OD}_{600}$ of 0.2 . The initial glucose concentration in either YPD medium or SC medium was $40 \mathrm{~g} / \mathrm{L}$. Before cultivation, $10 \mathrm{~g} / \mathrm{L}$ D-galactose was supplemented into the media to induce the gene expressions controlled by GAL promoters. Yeast cells were harvested after 100-h growth.

Fed-batch fermentation was conducted in YPD medium supplemented with $20 \mathrm{~g} / \mathrm{L}$ glucose as the initial carbon source. Seed cultures were obtained via overnight culture from an $\mathrm{OD}_{600}$ of $0.2-8.0$ in YPD medium. Then $200 \mathrm{~mL}$ seed cultures were transferred into a 5-L bioreactor (BLBIO-5GJG-2, Shanghai, China) with an initial OD600 of 0.8. Fermentation was performed in 2-L cultures at $30{ }^{\circ} \mathrm{C} . \mathrm{PH}$ and air flow were controlled at 5.8 and $1 \mathrm{vvm}$, respectively. The dissolved oxygen (DO) was maintained around $40 \%$ through adjusting the agitation speed. $50 \%(\mathrm{v} / \mathrm{v})$ glucose was fed periodically into the culture to keep the glucose concentration under $2 \mathrm{~g} / \mathrm{L}$. When cells entered post-log phase $\left(\mathrm{OD}_{600}\right.$ around 140), glucose feeding was stopped, and galactose solution was added into the bioreactor with a final concentration of $20 \mathrm{~g} / \mathrm{L}$. At the same time, ethanol was fed into the culture until the end of the fermentation. At least independent duplicate samples were collected to determine the cell density, glucose concentration, ethanol concentration, and 7-DHC production [7].

\section{Protein expression quantification}

In order to determine the expression levels of DHCR24s employed in this study, polyhistidine-tag was attached to the C-terminal of each tested DHCR24 in its particular expression cassette by PCR amplification with the primers listed in Table S1. Then the cassette was integrated into chromosome of strain syBE_Sc0125XJ01 with the same procedure to generate strain SyBE_Sc01250050 (obtaining strain SyBE_Sc0125H001-03/05/07/09/10-13/50, Table 1). These strains were grown in YPD medium for $40 \mathrm{~h}$ (ethanol consumption phase). The protein extraction and western-blot were then conducted according to Kinzurik et al. [49] and Rodriguez-Escudero et al. [50]. To be specific, $0.5 \mathrm{~mL}$ cells $\left(\mathrm{OD}_{600}\right.$ around 20$)$ were 
harvested and resuspended in $200 \mu \mathrm{L} 0.1 \mathrm{M} \mathrm{NaOH}$ for 5 min incubation at room temperature. Then cell pellets were harvested, resuspended in $50 \mu \mathrm{L}$ SDS sample buffer (60 mM Tris-HCl (pH 6.8), 5\% glycerol, 2\% SDS, $4 \% \beta$-mercaptoethanol, $0.0025 \%$ bromophenol blue), and boiled for $10 \mathrm{~min} .20 \mu \mathrm{L}$ cell lysis were loaded onto 10\% SDS-PAGE gel. After electrophorescence, proteins were transferred to PVDF membranes. Membranes were blocked with 5\% BSA in TBST buffer $(10 \mathrm{mM}$ Tris $(\mathrm{pH}$ 8.0), $150 \mathrm{mM} \mathrm{NaCl}, 0.05 \%$ Tween 20), then incubated with primary anti-polyhistidine (1:2000, Rayantibody RM1001, China), or anti-GAPDH (HRP) (1:5000, Abcam ab9385, UK) overnight at $4{ }^{\circ} \mathrm{C}$ with shaking. Afterward, membranes were repeatedly washed with TBST buffer. The membrane probed to anti-polyhistidine required further incubation with secondary HRP-conjugated goat anti-mouse antibody (Rayantibody, China). Signals were detected following the of SuperSignal ${ }^{\mathrm{TM}}$ West Pico PLUS Chemiluminescent Substrate Kit (Thermo, USA) by using Azure Biosystems C280 Chemiluminescent Blot Imaging System (USA). The intensities of the bands in westernblot pictures were quantified with Quantity One (Biorad, USA). The relative expression level of each DHCR24 was determined as the gray scale of anti-polyhistidine band divided by that of anti-GAPDH.

\section{Extraction and analysis of sterols}

Extraction and analysis of sterols were applied according to $\mathrm{Su}$ et al. [7] with some modification. Yeast cells were harvested by $12,000 \mathrm{rpm}$ centrifugation and resuspended by $3 \mathrm{~N} \mathrm{HCl}$. The suspension was boiled for $5 \mathrm{~min}$, and then the cells debris was washed by distilled water until $\mathrm{pH}$ was neutral. Just in case, $\mathrm{NaOH}$ solution was used to neutralize the residual $\mathrm{HCl}$. The cell pellet was resuspended by $1.5 \mathrm{M} \mathrm{NaOH}$-methonal solution and incubated at $60{ }^{\circ} \mathrm{C}$ for $4 \mathrm{~h}$. Then $\mathrm{n}$-hexane was added for sterols extraction with vortex. After centrifugation, the $\mathrm{n}$-hexane phase was collected and dried by centrifugal vacuum evaporator. Derivatization of the dried products was conducted with $N$-methyl- $N$-(trimethylsilyl) trifluoroacetamide (MSTFA) at $30{ }^{\circ} \mathrm{C}$ for $2 \mathrm{~h}$ to gain the sample ready for analysis.

The sterols were separated on an Agilent 6890 gas chromatograph (GC) (USA) coupled to Waters time-of-flight mass spectrometry (TOF-MS) (USA). The gas chromatograph was equipped with a DB-5 fused-silica capillary column $(30 \mathrm{~m} \times 0.25 \mathrm{~mm}$ i.d., film thickness $0.25 \mu \mathrm{m}$, J\&W Scientific, CA). Ions were generated by a $70 \mathrm{eV}$ electron beam in EI mode at an ionization current of $40 \mu \mathrm{A}$. Mass spectra were acquired in a range of $50-800 \mathrm{~m} / z$. The ion source temperature was $250{ }^{\circ} \mathrm{C}$, and the injection site temperature was $260{ }^{\circ} \mathrm{C}$. The temperature was initially $70{ }^{\circ} \mathrm{C}$ for $2 \mathrm{~min}$, then it was increased at $30{ }^{\circ} \mathrm{C} / \mathrm{min}$ to $250{ }^{\circ} \mathrm{C}$, and finally followed by an increase to $280{ }^{\circ} \mathrm{C}$ at $10{ }^{\circ} \mathrm{C} / \mathrm{min} .280^{\circ} \mathrm{C}$ was kept for $15 \mathrm{~min}$, and was increased to the final temperature $290{ }^{\circ} \mathrm{C}$ at $5{ }^{\circ} \mathrm{C} / \mathrm{min}$. The final temperature was maintained for $5 \mathrm{~min}$. Sterol standards (squalene, lanosterol, zymosterol, and 7-DHC) were purchased from Sigma-Aldrich (USA).

\section{Genes transcriptional analysis}

Transcription levels of genes in 7-DHC biosynthesis pathway were analyzed by Real-Time PCR. Strains were cultured in shake flask for $10 \mathrm{~h}$ (glucose consumption phase) and $30 \mathrm{~h}$ (ethanol consumption phase), respectively, and then harvested. Total RNA extraction, reverse transcription, and quantitative PCR were carried out by Apexbio Inc. (China) based on Wang et al. [51]. The relative transcription level for each gene was determined by $2^{-\Delta \Delta \mathrm{Ct}}$ method [52]. Gene ALG9 was used for normalization [53]. All data were from at least triplicate experiments. The statistical analysis ( $T$ test) was conducted using the SPSS 19.0 package to demonstrate variations between the tested groups. The level of significance was set at $P<0.05$.

\section{Additional file}

Additional file 1: Table S1. Oligonucleotides used in this study. Table S2. The Codon-optimized sequences of DHCR24s involved in this study. Table S3 Plasmids used in this study. Figure S1. Relative transcription level of the MVA pathway genes ( $\mathrm{a}-\mathrm{h}$ ) and the post squalene pathway genes $(\mathrm{i}-\mathrm{r}$ ) in control and $\triangle E R G 5$ strain. Cells were harvested at $10 \mathrm{~h}$ (glucose consumption phase) and $30 \mathrm{~h}$ (ethanol consumption phase). The relative transcription level for each gene was determined as $2^{-\Delta \Delta C t}$ using gene ALG9 for normalization. All data were from at least triplicate experiments. Significance levels of t-test were determined as "*" is for $P<0.05$ and "**" is for $P<0.01$. Figure $\mathbf{S 2}$. Cell growths of strains with $\mathrm{DHCR} 24 \mathrm{~s}$ from diversity species in YPD medium. The error bars represent standard deviation calculated from triplicate experiments. Hs, Homo sapiens; Mm, Mus musculus; Dr, Danio rerio; Ec, Equus caballus; Gg, Gallus gallus; Xt, Xenopus tropicalis; Bt, Bos Taurus; At, Arabidopsis thaliana; Gh, Gossypium hirsutum; Cg, Cryptococcus gattii; Tg, Trypanosoma grayi. Figure S3. Cell growths of strains SyBE_Sc01250009, SyBE_Sc0125XJ02 and SyBE_Sc0125XJ03 in YPD medium. Figure S4. Cell growths of strains SyBE_Sc0125XJ03, SyBE_Sc0125XJ04 and SyBE_Sc0125XJ06 in YPD medium. Figure S5. Effect of deleting lipids metabolism associated gene(s) on biomass building-up and 7-DHC production. a Cell growths of strains SyBE_Sc0125XJ06, SyBE_Sc0125XJ07, SyBE_Sc0125XJ08 and SyBE_Sc0125XJ09 in YPD medium as well as that of SyBE_Sc0125XJ08 in SC medium. b 7-DHC production of strain SyBE_Sc0125XJ08 in YPD medium and SC medium.

\section{Abbreviations}

7-DHC: 7-dehydrocholesterol; Hs: Homo sapiens; Mm: Mus musculus; Dr: Danio rerio; Ec: Equus caballus; Gg: Gallus gallus; Xt: Xenopus tropicalis; Bt: Bos taurus; At: Arabidopsis thaliana; Gh: Gossypium hirsutum; Cg: Cryptococcus gattii; Tg: Trypanosoma grayi; OE-PCR: overlap extension PCR; MVA: mevalonate; MSTFA: $\mathrm{N}$-methyl-N-(trimethylsilyl) trifluoroacetamide; GC-TOF-MS: gas chromatography time-of-flight mass spectrometry; DHCR24: $\triangle^{24}$-dehydrocholesterol reductase; FLD1: few lipid droplets gene1; PAH1: phosphatidate phosphatase; DO: dissolved oxygen. 


\section{Authors' contributions}

XJG and YJY conceived of the study. XJG and BXZ participated in strain construction. MY carried out the protein analysis. XJG and YW carried out the molecular genetic studies. XJG and WHX participated in fed-batch fermentation. XJG and HL carried out sterols analysis. YW, WHX, and MDY participated in design and coordination of the study as well as helped to draft the manuscript. GRZ helped to revise the manuscript. YJY supervised the whole research and revised the manuscript. All authors read and approved the final manuscript.

\section{Author details}

${ }^{1}$ Key Laboratory of Systems Bioengineering (Ministry of Education), School of Chemical \& Engineering, Tianjin University, No. 92, Weijin Road, Nankai District, Tianjin 300072, People's Republic of China. ${ }^{2}$ SynBio Research Platform, Collaborative Innovation Center of Chemical Science and Engineering (Tianjin), Tianjin University, Tianjin 300072, People's Republic of China.

\section{Acknowledgements}

The authors gratefully acknowledge the financial support from the National Natural Science Foundation of China (21621004 and 31570088), the Ministry of Science and Technology of China ("973" Program: 2014CB745100), and the Innovative Talents and Platform Program of Tianjin (16PTSYJC00050 and 16PTGCCX00140).

\section{Competing interests}

The authors declare that they have no competing interests.

\section{Availability of supporting data}

Data will be made available from the corresponding author on reasonable request.

\section{Consent for publication}

All authors read and approved the final manuscript.

\section{Ethical approval and consent to participate}

Not applicable.

\section{Funding}

The authors gratefully acknowledge the financial support from the National Natural Science Foundation of China (21621004 and 31570088), the Ministry of Science and Technology of China ("973" Program: 2014CB745100), and the Innovative Talents and Platform Program of Tianjin (16PTSYJC00050 and 16PTGC(X00140).

\section{Publisher's Note}

Springer Nature remains neutral with regard to jurisdictional claims in published maps and institutional affiliations.

Received: 26 February 2018 Accepted: 6 July 2018

Published online: 16 July 2018

\section{References}

1. Wolf G. The discovery of vitamin D: the contribution of Adolf Windaus. J Nutr. 2004;134:1299-302.

2. Bendik I, Friedel A, Roos FF, Weber P, Eggersdorfer M. Vitamin D: a critical and essential micronutrient for human health. Front Physiol. 2014;5:248.

3. Pilz S, Trummer C, Pandis M, Schwetz V, Aberer F, Grubler M, Verheyen N, Tomaschitz A, Marz W. Vitamin D: current guidelines and future outlook. Anticancer Res. 2018;38:1145-51.

4. Van Schoor N, Lips P. Global overview of vitamin D status. Endocrinol Metab Clin North Am. 2017:46:845-70

5. Lang C, Markus V. Preparation of 7-dehydrocholesterol and/or the biosynthetic intermediates and/or secondary products thereof in transgenic organisms. US Patent 12607017; 2011.

6. Hohmann HP, Lehmann M, Merkamm M. Production of non-yeast sterols by yeast. US Patent, 20120231495; 2012.
7. Su W, Xiao WH, Wang Y, Liu D, Zhou X, Yuan YJ. Alleviating redox imbalance enhances 7-dehydrocholesterol production in engineered Saccharomyces cerevisiae. PLoS ONE. 2015;10:e0130840.

8. Nielsen J, Keasling JD. Engineering cellular metabolism. Cell. 2016:164:1185-97.

9. Shin $\mathrm{GH}$, Veen $\mathrm{M}$, Stahl U, Lang C. Overexpression of genes of the fatty acid biosynthetic pathway leads to accumulation of sterols in Saccharomyces cerevisiae. Yeast. 2012;29:371-83.

10. Fei W, Shui G, Gaeta B, Du X, Kuerschner L, Li P, Brown AJ, Wenk MR, Parton $\mathrm{RG}$, Yang $\mathrm{H}$. Fld 1 p, a functional homologue of human seipin, regulates the size of lipid droplets in yeast. J Cell Biol. 2008;180:473-82.

11. Park Y, Han GS, Mileykovskaya E, Garrett TA, Carman GM. Altered lipid synthesis by lack of yeast pah1 phosphatidate phosphatase reduces chronological life span. J Biol Chem. 2015;290:25382-94.

12. Keasling JD. Manufacturing molecules through metabolic engineering. Science. 2010;330:1355-8.

13. Dahl RH, Zhang F, Alonso-Gutierrez J, Baidoo E, Batth TS, Redding-Johanson AM, Petzold CJ, Mukhopadhyay A, Lee TS, Adams PD, Keasling JD. Engineering dynamic pathway regulation using stress-response promoters. Nat Biotechnol. 2013;31:1039-46.

14. Jacquier N, Schneiter R. Mechanisms of sterol uptake and transport in yeast. J Steroid Biochem Mol Biol. 2012;129:70-8.

15. Wang GS, Grammel H, Abou-Aisha K, Sagesser R, Ghosh R. High-level production of the industrial product lycopene by the photosynthetic bacterium Rhodospirillum rubrum. Appl Environ Microbiol. 2012;78:7205-15.

16. Souza CM, Schwabe TM, Pichler H, Ploier B, Leitner E, Guan XL, Wenk MR, Riezman I, Riezman H. A stable yeast strain efficiently producing cholesterol instead of ergosterol is functional for tryptophan uptake, but not weak organic acid resistance. Metab Eng. 2011;13:555-69.

17. Westfall PJ, Pitera DJ, Lenihan JR, Eng D, Woolard FX, Regentin R, Horning $\mathrm{T}$, Tsuruta $\mathrm{H}$, Melis DJ, Owens A, et al. Production of amorphadiene in yeast, and its conversion to dihydroartemisinic acid, precursor to the antimalarial agent artemisinin. Proc Natl Acad Sci USA. 2012;109:E111-8.

18. Du HX, Xiao WH, Wang Y, Zhou X, Zhang Y, Liu D, Yuan YJ. Engineering Yarrowia lipolytica for campesterol overproduction. PLoS ONE. 2016:11:e0146773.

19. Yang $\mathrm{H}$, Tong J, Lee CW, Ha S, Eom SH, Im YJ. Structural mechanism of ergosterol regulation by fungal sterol transcription factor Upc2. Nat Commun. 2015;6:6129.

20. Davies BS, Wang HS, Rine J. Dual activators of the sterol biosynthetic pathway of Saccharomyces cerevisiae: similar activation/ regulatory domains but different response mechanisms. Mol Cell Biol. 2005:25:7375-85.

21. Lu X, Li Y, Liu J, Cao X, Wang X, Wang D, Seo H, Gao B. The membrane topological analysis of 3beta-hydroxysteroid-Delta24 reductase (DHCR24) on endoplasmic reticulum. J Mol Endocrinol. 2012;48:1-9.

22. Sarria S, Wong B, Garcia Martin H, Keasling JD, Peralta-Yahya P. Microbial synthesis of pinene. ACS Synth Biol. 2014;3:466-75.

23. Zhang Y, Wang Y, Yao M, Liu H, Zhou X, Xiao W, Yuan Y. Improved campesterol production in engineered Yarrowia lipolytica strains. Biotechnol Lett. 2017:39:1033-9.

24. Chen Y, Xiao W, Wang Y, Liu H, Li X, Yuan Y. Lycopene overproduction in Saccharomyces cerevisiae through combining pathway engineering with host engineering. Microb Cell Fact. 2016;15:113.

25. Waterham HR, Koster J, Romeijn GJ, Hennekam RC, Vreken P, Andersson HC, FitzPatrick DR, Kelley RI, Wanders RJ. Mutations in the $3 \beta$-hydroxysterol $\Delta^{24}$-reductase gene cause desmosterolosis, an autosomal recessive disorder of cholesterol biosynthesis. Am J Hum Genet. 2001;69:685-94.

26. Strausberg RL, Feingold EA, Grouse LH, Derge JG, Klausner RD, Collins FS, Wagner L, Shenmen CM, Schuler GD, Altschul SF, et al. Generation and initial analysis of more than 15,000 full-length human and mouse cDNA sequences. Proc Natl Acad Sci USA. 2002;99:16899-903.

27. Hestand MS, Kalbfleisch TS, Coleman SJ, Zeng Z, Liu J, Orlando L, MacLeod JN. Annotation of the protein coding regions of the equine genome. PLoS ONE. 2015:10:e0124375.

28. Caldwell RB, Kierzek AM, Arakawa H, Bezzubov Y, Zaim J, Fiedler P, Kutter S, Blagodatski A, Kostovska D, Koter M, et al. Full-length cDNAs from chicken bursal lymphocytes to facilitate gene function analysis. Genome Biol. 2005;6:R6. 
29. Klein SL, Strausberg RL, Wagner L, Pontius J, Clifton SW, Richardson P. Genetic and genomic tools for Xenopus research: the NIH Xenopus initiative. Dev Dyn. 2002;225:384-91.

30. Zerenturk EJ, Sharpe LJ, Ikonen E, Brown AJ. Desmosterol and DHCR24: unexpected new directions for a terminal step in cholesterol synthesis. Prog Lipid Res. 2013;52:666-80.

31. Klahre U, Noguchi T, Fujioka S, Takatsuto S, Yokota T, Nomura T, Yoshida S, Chua NH. The Arabidopsis DIMINUTO/DWARF1 gene encodes a protein involved in steroid synthesis. Plant Cell. 1998;10:1677-90.

32. Kelly S, Ivens A, Manna PT, Gibson W, Field MC. A draft genome for the African crocodilian trypanosome Trypanosoma grayi. Sci Data. 2014;1:140024.

33. D'Souza CA, Kronstad JW, Taylor G, Warren R, Yuen M, Hu G, Jung WH, Sham A, Kidd SE, Tangen K, et al. Genome variation in Cryptococcus gattii, an emerging pathogen of immunocompetent hosts. MBio. 2011;2:e00342-10

34. Peng B, Plan MR, Carpenter A, Nielsen LK, Vickers CE. Coupling gene regulatory patterns to bioprocess conditions to optimize synthetic metabolic modules for improved sesquiterpene production in yeast. Biotechnol Biofuels. 2017;10:43.

35. Wu XL, Li BZ, Zhang WZ, Song K, Qi H, Dai JB, Yuan YJ. Genome-wide landscape of position effects on heterogeneous gene expression in Saccharomyces cerevisiae. Biotechnol Biofuels. 2017;10:189.

36. Siniossoglou S. Phospholipid metabolism and nuclear function: roles of the lipin family of phosphatidic acid phosphatases. Biochim Biophys Acta. 2013;1831:575-81.

37. Rowlett WW, Mallampalli V, Karlstaedt A, Dowhan W, Taegtmeyer H, Margolin W, Vitrac H. Impact of membrane phospholipid alterations in Escherichia coli on cellular function and bacterial stress adaptation. J Bacteriol. 2017;199:e00849-16.

38. Veen M, Stahl U, Lang C. Combined overexpression of genes of the ergosterol biosynthetic pathway leads to accumulation of sterols in Saccharomyces cerevisiae. FEMS Yeast Res. 2003;4:87-95.

39. Arendt P, Miettinen K, Pollier J, De Rycke R, Callewaert N, Goossens A. An endoplasmic reticulum-engineered yeast platform for overproduction of triterpenoids. Metab Eng. 2017:40:165-75.

40. Ott RG, Athenstaedt K, Hrastnik C, Leitner E, Bergler H, Daum G. Flux of sterol intermediates in a yeast strain deleted of the lanosterol C-14 demethylase Erg11 p. Biochim Biophys Acta. 2005;1735:111-8.
41. Shobayashi M, Mitsueda S, Ago M, Fujii T, Iwashita K, lefuji H. Effects of culture conditions on ergosterol biosynthesis by Saccharomyces cerevisiae. Biosci Biotechnol Biochem. 2005;69:2381-8.

42. Parks LW, Casey WM. Physiological implications of sterol biosynthesis in yeast. Annu Rev Microbiol. 1995;49:95-116.

43. Polakowski T, Bastl R, Stahl U, Lang C. Enhanced sterol-acyl transferase activity promotes sterol accumulation in Saccharomyces cerevisiae. App Microbiol Biotechnol. 1999;53:30-5.

44. Sandager L, Dahlqvist A, Banas A, Stahl U, Lenman M, Gustavsson M Stymne S. An acyl-CoA:cholesterol acyltransferase (ACAT)-related gene is involved in the accumulation of triacylglycerols in Saccharomyces cerevisiae. Biochem Soc Trans. 2000;28:700-2.

45. Hohmann HP, Leber R, Lehmann M, Odar C, Petschacher B, Pichler H, Ploier B. Production of sterols in modified yeast. US patent 2016081936; 2017.

46. Gietz RD. Yeast transformation by the LiAc/SS carrier DNA/PEG method Methods Mol Biol. 2014;1163:33-44.

47. Kumar S, Stecher G, Tamura K. MEGA7: molecular evolutionary genetics analysis version 7.0 for bigger datasets. Mol Biol Evol. 2016;33:1870-4.

48. Gibson DG, Smith HO, Hutchison CA 3rd, Venter JC, Merryman C. Chemical synthesis of the mouse mitochondrial genome. Nat Methods. 2010;7:901-3.

49. Kinzurik MI, Ly K, David KM, Gardner RC, Fedrizzi B. The GLO1 gene is required for full activity of o-acetyl homoserine sulfhydrylase encoded by MET17. ACS Chem Biol. 2017:12:414-21.

50. Rodriguez-Escudero I, Fernandez-Acero T, Cid VJ, Molina M. Heterologous mammalian Akt disrupts plasma membrane homeostasis by taking over TORC2 signaling in Saccharomyces cerevisiae. Sci Rep. 2018;8:7732.

51. Wang R, Gu X, Yao M, Pan C, Liu H, Xiao W, Wang Y, Yuan Y. Engineering of $\beta$-carotene hydroxylase and ketolase for astaxanthin overproduction in Saccharomyces cerevisiae. Front Chem Sci Eng. 2017;11:89-99.

52. Livak KJ, Schmittgen TD. Analysis of relative gene expression data using real-time quantitative PCR and the $2^{-\triangle A C T}$ method. Methods. 2001;25:402-8.

53. Stovicek V, Borja GM, Forster J, Borodina I. EasyClone 2.0: expanded toolkit of integrative vectors for stable gene expression in industrial Saccharomyces cerevisiae strains. J Ind Microbiol Biotechnol. 2015;42:1519-31.
Ready to submit your research? Choose BMC and benefit from:

- fast, convenient online submission

- thorough peer review by experienced researchers in your field

- rapid publication on acceptance

- support for research data, including large and complex data types

- gold Open Access which fosters wider collaboration and increased citations

- maximum visibility for your research: over $100 \mathrm{M}$ website views per year

At $\mathrm{BMC}$, research is always in progress.

Learn more biomedcentral.com/submissions 